\title{
Nasopharyngeal maltoma: An intriguing case report
}

\author{
Ajmal Sherif \\ 1Consultant, Dept. of Medical Oncology, MVR Cancer Center and Research Institute, Kerala, India \\ *Corresponding Author: Ajmal Sherif \\ Email: drajmal@mvrccri.co
}

\begin{abstract}
Non-Hodgkin's lymphoma (NHL) of Nasopharynx accounts for $8 \%$ of all the tumours affecting the region. It creates a dilemma for the clinician as well as the pathologist. Maltoma (MALT) of Nasopharynx is a low grade lymphoma which is generally slow growing in nature. Biopsy and immunohistochemistry is vital in diagnosis of this disease. However it is capable of causing cranial nerve palsy by local invasion. A high degree of suspicion is needed for the clinician to accurately diagnose this rare condition
\end{abstract}

Keywords: Nasopharyngeal Lymphoma, Maltoma, Immunohistochemistry.

\section{Introduction}

Lymphomas are neoplasms that arise from the white blood cells and are divided into two large subtypes: Hodgkin and non-Hodgkin lymphoma. B-cell lymphoma constitutes the most common subtype of non-Hodgkin lymphoma; almost $85 \%$ of patients with lymphoma have this variant. Lymphomas represent the second malignant head and neck tumours region after squamous cell carcinoma. ${ }^{1}$ It represents $2-2.5 \%$ of head and neck tumours. ${ }^{2}$ In NHL, the primary site of origin is extranodal in $40 \%$ of cases ${ }^{3}$ Lymphomas can arise from any lymphoid tissue located in the body; however, primary Nasopharyngeal Non-Hodgkin lymphoma(NPNHL) is extremely rare. Among nasopharyngeal Non Hodgkin Lymphoma, Maltoma are rarely encountered in clinical practice. We report a rare case report of a 64-year-old man diagnosed with Primary Nasopharyngeal Maltoma.

\section{Case Report}

A 64 year old gentleman nonsmoker with ECOG PS 3 came with complaints of headache, cough and regurgitation while eating food for one month duration.There was history of loss of appetite and weight loss of $3 \mathrm{~kg}$ in the preceding 3 months. On Examination he was found to have features of multiple cranial nerve palsies. He had features of Right upper quadrantanopia which implied a left temporal lesion, Conductive hearing loss of left side (CN VIII palsy), deviation of uvula to right side (CN X palsy) and reduced gag reflex (CN IX palsy).His motor and sensory system examination of the limbs were within normal limits. No history of fever, weight loss or night sweats.No organomegaly or adenopathy present. Other Systems were within normal limits

\section{MRI Neck}

Heterogeneously enhancing lesion in left superolateral prevertebral space (approximate size 35 x 28mm), appearing iso to hyperintense on T2WI and hypointense on T1WI. Lesion extends to the base of the skull and infiltrates left jugular foramen. Left internal jugular vein and internal carotid artery are encased by the lesion. There is extension of the lesion caudally to the left temporal lobe, laterally to the left mandibular condyle and left parapharyngeal space with erosion of the base of the skull.(Fig. A)

Direct nasal endoscopy was done and multiple punch biopsies were taken.Biopsy showed fragments of tissue lined by ciliated columnar epithelium with subepithelium showing a diffusely lying dense, monotonous population of small lymphoid cells with focal plasmacytoid differentiation (Fig. B). Lymphoepithelial lesions and residual germinal centers were present.

Immunohistochemistry was done which showed evidence of Maltoma.(Fig. C-G)

\begin{tabular}{|l|l|}
\hline Marker & Result \\
\hline PanCK & Negative \\
\hline CD20 & Diffuse strong positive \\
\hline CD3 & $\begin{array}{l}\text { Background T cells are } \\
\text { positive }\end{array}$ \\
\hline CD5 & $\begin{array}{l}\text { Background T cells are } \\
\text { positive }\end{array}$ \\
\hline Cyclin D1 & Negative \\
\hline Bc12 & Positive \\
\hline CD 10 & Negative \\
\hline Ki 67 & $10-15 \%$ \\
\hline
\end{tabular}




\section{FDG PET CT}

Intensely FDG avid heterogeneously enhancing soft tissue lesion in the posterosuperior nasopharynx with local extension No definite PET scan evidence of clinically significant abnormal hypermetabolism elsewhere in the body in the present study.

As the patient was unfit for chemotherapy, he was referred for local Radiotherapy. However the patient died of Myocardial Infarction before completion of treatment.

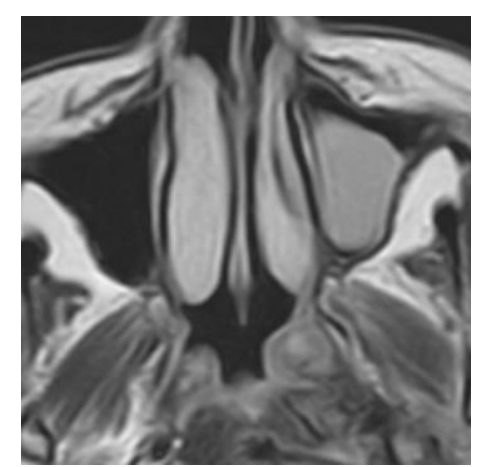

Fig A: CT showing nasopharyngeal mass (left side) with extension to nearby structures.

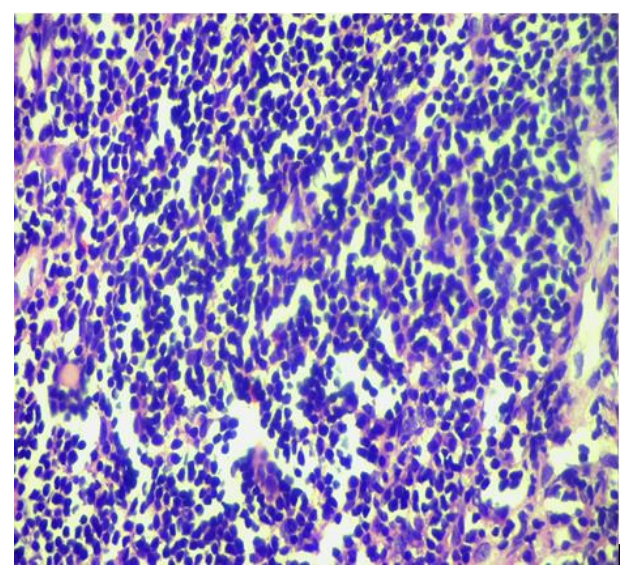

Fig. B: Histopathology, Clusters of Small lymphoid cells

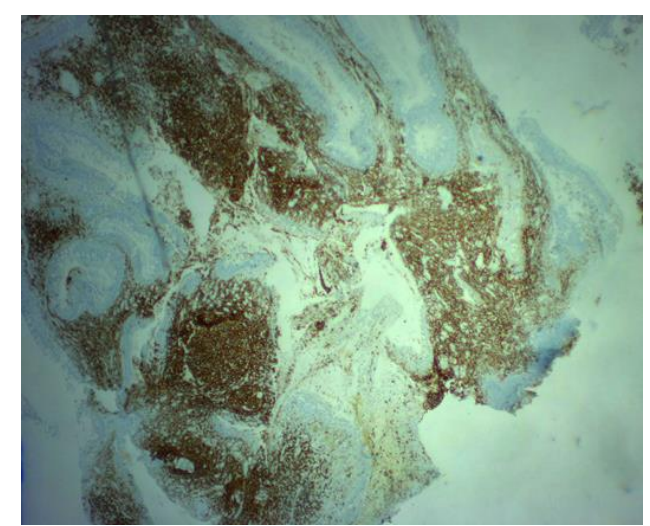

Fig. C: CD20: Diffuse strong positive

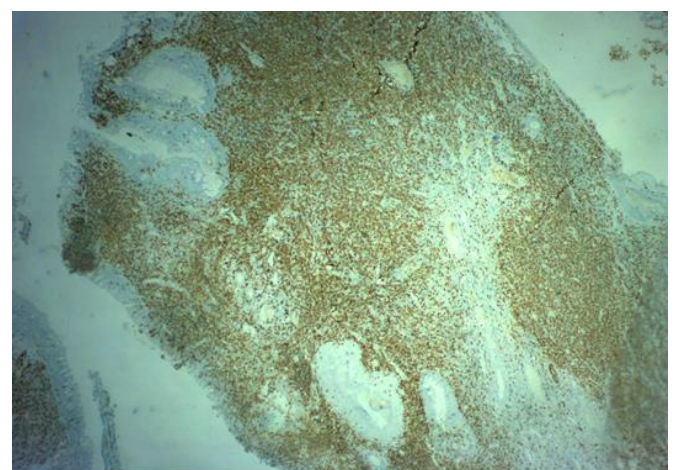

Fig. D: CD3: Negative

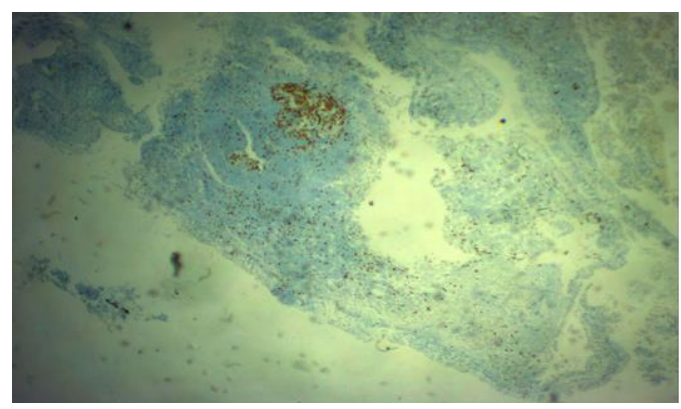

Fig. E: Ki67: Low

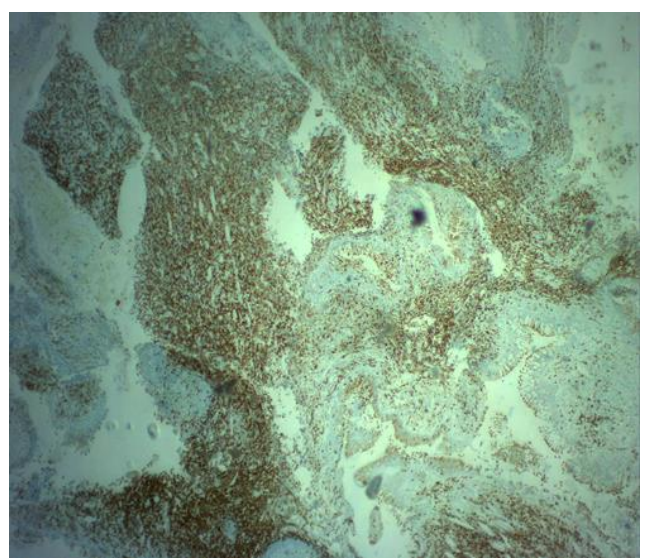

Fig. F: CD5: Negative

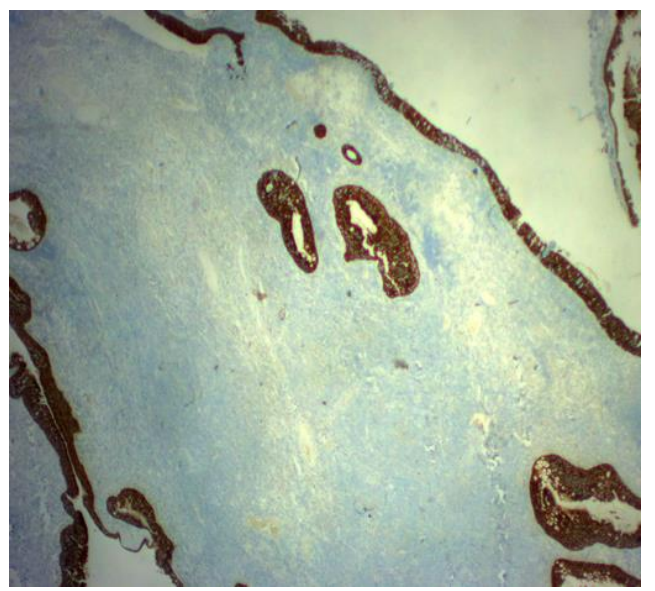

Fig. G: Cytokeratin K: Negative 


\section{Discussion}

NPNHL is a homogeneous tumour that tends to diffusely involve all walls of the nasopharynx and spread in an exophytic fashion to fill the airway, rather than infiltrating into the deep tissues.

The differential diagnosis of a nasopharyngeal tumours includes nasopharyngeal carcinoma (squamous cell or undifferentiated carcinoma), adenocarcinoma, adenoid cystic carcinoma, melanoma, plasmacytoma, sarcomas such a rhabdomyosarcoma, and metastases. ${ }^{4}$ On imaging, NHL should be considered as against nasopharyngeal carcinoma if there is a symmetric nasopharyngeal mass with marked homogeneity, a high degree of enhancement, and a higher Waldeyer ring involvement combined with no invasion into the deep structures. ${ }^{5}$ However it is a difficult case to diagnose, as lymphoid hyperplasia is often seen in this region. Immunohistochemistry can differentiate between the two entities. Usually Maltomas are positive for CD20 and negative for CD5, CD3 and CD43. ${ }^{6}$ Primary NHL more commonly spreads superficially to involve the nasal cavity or oropharynx, lymphadenopathy is frequent and extensive. ${ }^{7}$ The treatment options for MALT lymphomas include single agent Rituximab, low dose cyclophosphamide or even multiple chemotherapy regimens. Localized MALT lymphomas can be treated effectively with radiotherapy or surgery. ${ }^{8}$

Relapse usually occurs in the first 2 years and those with disease that failed to respond to primary treatment shows rapid progression of disease. Central nervous system relapse has been shown to be associated with head and neck NHL that are of intermediate and high-grade histology.

\section{Conclusion}

Nasopharyngeal Maltoma is infrequently encountered in routine clinical practice. Biopsy and Immunohistochemistry can clearly differentiate it from other types of malignancies. Multidisciplinary management is needed for the successful treatment of this condition.

\section{Conflicts of interest}

None.

\section{Financial support}

No grants or funds received

\section{References}

1. De Peña CA, Van Tassel P, Lee YY. Imaging Hodgkin and Non-Hodgkin Lymphoma in the Head and Neck. Radiol Clin North Am 1990; 28(4):723-43

2. Essadi I, Ismaili N, Tazi E, Elmajjaoui S, Saidi A, Ichou M: et al. Primary lymphoma of the head and neck: two case reports and review of the literature. Cases $J$ 2008;1:42610.1186/1757-1626-1-426.

3. Allam W, Ismaili N, Elmajjaoui S, Elgueddari BK, Ismaili M, Errihani H. et al. Primary Nasopharyngeal non-Hodgkin lymphomas: a retrospective review of 26 Moroccan patients. BMC Ear Nose Throat Disord 2009;9:11. Published 2009 Nov 17. doi:10.1186/1472-6815-9-11

4. Cho KS, Kang DW, Kim HJ, Lee JK, Roh HJ. Differential diagnosis of primary nasopharyngeal lymphoma and nasopharyngeal carcinoma focusing on CT, MRl, and PET/CT. Otolaryngol Head Neck Surg 2012;146:574-8.

5. King, A.D .Non-Hodgkin's Lymphoma of the Nasopharynx: CT and MR Imaging. Clin Radiol 58(8):6215.

6. Cheung MMC, Chan JKC, Lau WH. Primary NonHodgkin'slymphoma of the nose and nasopharynx: clinical features, tumor immunophenotype, and treatment outcome in 113 patients. J Clin Oncol 1998;16:70-7.

7. Ravikanth R, Kamalasekar K. Primary nasopharyngeal non-Hodgkin lymphoma. Int J Clinicopathol Correl 2019;3:39-40

8. Allam W. Ismaili N. Elmajjaoui. Primary Nasopharyngeal non-Hodgkin lymphomas: a retrospective review of 26 Moroccan patients. BMC Ear Nose Throat Disord 2009;9(11).

How to cite: Sherif A. Nasopharyngeal maltoma: An intriguing case report. IP Int J Med Paediatr Oncol 2020;6(1):35-7. 